\title{
COPD and PE: A clinical dilemma
}

\author{
Teng Moua' \\ Kenneth Wood ${ }^{2}$ \\ 'Department of Internal Medicine, \\ ${ }^{2}$ Section of Pulmonary and Critical \\ Care Medicine, University of \\ Wisconsin Hospitals and Clinics, \\ Madison, WI, USA
}

\begin{abstract}
Dyspnea in patients with known chronic obstructive pulmonary disease (COPD) can be a clinical challenge due to the nonspecific nature of atypical presentations. Typical features of fever, productive cough, and wheezing on presentation support COPD exacerbation, while absence of such findings may warrant further evaluation for underlying etiologies, including pulmonary embolism (PE). It is suspected that one in four patients with atypical COPD exacerbation may have $\mathrm{PE}$ as an underlying or concomitant cause of acute dyspnea. This review discusses the clinical presentation of COPD and PE, and presents an overview of the rationale for pursuing work-up for thromboembolic disease in the setting of known obstructive lung diseases.

Keywords: pulmonary embolism, chronic obstructive pulmonary disease, COPD, dyspnea
\end{abstract}

\section{Introduction}

The chronic obstructive pulmonary disease (COPD) patient presenting with acute dyspnea can be a diagnostic challenge in both the outpatient and inpatient arenas. Many cardiopulmonary diseases, including acute pulmonary embolism (PE), are worsened or masked by the presence of COPD. While diagnostic tests such as B-type natriuretic peptide have led to differentiating dyspnea in heart failure from COPD exacerbation, there are no proven clinical criteria to help delineate acute pulmonary embolism from COPD. This is attributable to the overlap and nonspecificity of clinical features common to both diseases.

Guidelines presented by the World Health Organization (WHO) and Global Initiative for Chronic Obstructive Lung Disease (GOLD) for the diagnosis of COPD stress the chronicity of dyspnea with exposure to relevant risk factors (GOLD et al 2005). Further diagnostic evaluation includes pulmonary function testing that reveals obstruction and supportive findings on chest X-ray (CXR). Acute exacerbation is diagnosed by clinical means and presents with a worsening of dyspnea with or without sputum change, a decline in exercise tolerance, and exam findings of air trapping and poor lung aeration. The progression usually takes a few days and does not present acutely although the patient may be in respiratory distress at the time of evaluation.

The presentation of pulmonary embolism is similarly subtle with nonspecific clinical features such as acute dyspnea, tachycardia, and pleuritic chest pain. While COPD remains a clinical diagnosis, PE requires objective confirmation of clot by an imaging study to warrant appropriate anticoagulation therapy. When recognizing that the mortality of untreated PE may be as high as $25 \%$, it is crucial to incorporate PE into the differential diagnosis of a COPD exacerbation, and instructive to review the incidence of PE in COPD patients in the reported literature.

\section{Prevalence and risk of PE with COPD}

Thromboembolism represents a spectrum of disease, ranging from deep venous thrombosis (DVT) to PE and infarct. The incidence of DVT is 160 per 100,000 persons, with 44\% developing either symptomatic or asymptomatic PE (Kummer 1998). 
The estimated annual incidence of pulmonary embolism is $23-69$ cases per 100,000 persons with hospitalizations resulting in 676,700 inpatient days (Kozak et al 2005). Approximately 187,000 new cases occur each year in individuals older than 45 , half of which are considered idiopathic (Cushman et al 2004). A compilation of studies examining the incidence of DVT in patients with COPD reported a rate of $10 \%-12 \%$, with the majority discovered by surveillance studies and clinically asymptomatic (Prescott et al 1981; Erelel et al 2002; Ambrosetti et al 2003). Autopsy studies have reported the incidence of PE in patients with COPD to be $28 \%-51 \%$ without defining the percentage of suspected antemortem diagnoses (Prescott et al 1981). Retrospective work by Mispelaere and colleagues (2002) reported a $20 \%$ prevalence of $\mathrm{PE}$ in 50 patients presenting with COPD exacerbation of noninfectious etiology and a prospective study of 31 patients by the same investigators found a prevalence of 29\% (9 of 31 patients). Tillie-Leblond and colleagues (2006) similarly reported $\mathrm{PE}$ in $25 \%$ of patients presenting with noninfectious COPD exacerbation. Using the Geneva score, three clinical features were statistically associated with PE; 1) prior history of malignancy, 2) history of DVT or PE, and 3) drop in $\mathrm{PaCO}_{2}$ greater than $5 \mathrm{mmHg}$. Lower extremity Doppler studies confirmed DVT in $51 \%$ of patients (TillieLeblond et al 2006). However, Rutschmann and colleagues (2007) found only 4 cases of computed tomography (CT) proven PE out of 123 consecutive patients presenting with COPD exacerbation, representing a prevalence of $3.3 \%$. As further studies are pursued, it is likely the prevalence of COPD-associated PE will become more apparent, making it important to include thromboembolism in the differential for COPD patients presenting with acute dyspnea.

The prevalence of PE in patients with COPD is important because of combined morbidity and mortality. In a followup of 1487 patients from the Prospective Investigation of Pulmonary Embolism Diagnosis (PIOPED) study, Carson and colleagues (1996) found an adjusted estimated relative risk of death at one year with COPD and PE of 1.94, compared with 1.1 for patients with $\mathrm{PE}$ alone. The one year mortality of those with COPD and PE was 53.3\%, in contrast to $15 \%$ of those with PE alone (Carson et al 1996).

\section{The clinical evaluation}

The clinical difficulty of diagnosing PE in the setting of COPD was described by Sharma and Sasahara (1975). Nonspecific findings such as pleuritic pain, tachypnea, tachycardia, and fever were common presentations of the two diseases
(Sharma and Sasahara 1975). It is valuable to review the presenting features of each to appreciate their overlap.

\section{Physical exam}

In 1819, Laennec recorded the first clinical descriptions of COPD as dyspnea, hyperresonant chest wall, diminished breath sounds, and wheezing. Contemporarily, classic exam findings of barrel chest with hyperinflated lungs, pursed lip breathing and accessory muscle use continue to signify airway obstruction. McGee (2001) reported 3 findings that strongly support the diagnosis of COPD exacerbation: early inspiratory crackles, absence of cardiac dullness on percussion, and decreased breath sounds. Absence of cardiac dullness over the left lower sternal border had a sensitivity of $15 \%$, but specificity of $99 \%$, while unforced wheeze was only $13 \%-56 \%$ sensitive and $86 \%-99 \%$ specific. Other clinical features of COPD exacerbation include productive cough secondary to an inciting respiratory infection, decreased exercise tolerance, and a breath - sound score of less than 9 (a scoring system of listening to over 6 locations on the chest wall, and then rating and totaling breath sounds at each site from 0 [absent] to 4 [louder than normal]). McGee (2001) suggested clinically combining a historical picture of 70 or more pack-years of smoking, prior diagnosis of COPD, and decreased breath sounds to gain a likelihood ratio of 25.7 for active COPD exacerbation.

Stein's evaluation of patients presenting with acute PE found dyspnea and tachypnea in $73 \%$ of patients, tachycardia in $30 \%$, and pleuritic pain in $66 \%$ (Stein et al 1991). Rales were heard in approximately half of patients. More specific findings in PE include hemoptysis, elevated neck veins, a loud P2 component of S2, and asymmetry in size of the lower extremities or calf tenderness. Of patients diagnosed with PE, only $45 \%$ had a presentation of pleuritic chest pain and hemoptysis (Pineda et al 2001). Other investigators noted only a history of cancer, asymmetric lower extremity swelling, or tachycardia as being reliable in arguing for a PE diagnosis, while a heart rate of less than 90 argued against it (McGee 2001). The reliability of lower extremity edema or asymmetry with calf pain (Homan's sign) concerning for DVT has fallen out of favor in recent years due to nonspecificity of the finding.

A review of the clinical features of each disease suggests appreciable overlap making differentiation difficult. For example, rales were prevalent in $58 \%$ of patients with $\mathrm{PE}$ while inspiratory crackles were $25 \%-77 \%$ sensitive and $98 \%$ specific in COPD (McGee 2001). Lesser et al found rales in $81 \%$ of patients with COPD and PE compared to $53 \%$ in 
COPD alone (Lesser et al 1992). Other overlapping features of COPD and PE on exam include tachypnea, tachycardia, and fever. Cough productive of sputum is less common in acute PE and more indicative of COPD exacerbation (Tillie-Leblond et al 2006; McGee 2001; Pineda et al 2001). Consequently, sub-acute PE may be overshadowed by or confound a bona fide COPD exacerbation, escaping suspicion for diagnosis on physical exam.

\section{Chest X-ray}

Findings supportive of COPD on CXR include increased lung volumes and hyperinflation. The presence of diffusely diminished lung markings suggests obliteration of supportive bronchial structures and correlates well with COPD; however, the CXR is otherwise nonspecific.

Stein's evaluation of chest radiographs in 117 patients diagnosed with PE and no prior lung disease found $68 \%$ had nonspecific parenchymal abnormalities. The majority of findings were atelectasis and small pleural effusions (Stein et al 1991). Classic features of PE on CXR, such as Westermark's sign or Hampton's hump, had poor sensitivity in the setting of COPD (Sharma and Sasahara 1975).

The CXR is of value for the evaluation of other acute causes of dyspnea including pneumonia, pleural effusion, pulmonary edema, or pneumothorax. The acutely dyspneic patient with minimal parenchymal findings points towards vascular or bronchial etiologies such as asthma, COPD exacerbation, or thromboembolic disease.

\section{Arterial blood gas}

Arterial blood gas findings in COPD are nonspecific, but are characterized by hypoxemia and hypercapnea, with elevated bicarbonate indicative of chronic metabolic correction of respiratory acidosis. In the acute setting, hypercapnea may be increased secondary to worsened deadspace ventilation and ventilation/perfusion (V/Q) mismatch caused by increased mucus production, bronchial constriction, or worsened alveolar-capillary interface destruction with disease progression.

With pulmonary embolism, Stein and colleagues (1991) found the $\mathrm{PaO}_{2}$ to be less than $80 \mathrm{mmHg}$ in $74 \%$ of patients without underlying pulmonary disease. The A-a gradient was greater than $20 \mathrm{mmHg}$ in $76 \%$ of patients (Stein et al 1991). $\mathrm{PaCO}_{2}$ was commonly lower due to tachypnea, except in the presence of increased deadspace ventilation. In a case report of 3 patients with known COPD complicated by PE, Lipmann and Fein (1981) suggested a drop in $\mathrm{PaCO}_{2}$ of $10 \mathrm{mmHg}$ or more in otherwise previously hypercapneic COPD patients as indicative of thromboembolism. The mean increase in
A-a gradient was $20( \pm 13) \mathrm{mmHg}$ with poor response to conventional bronchodilator therapy (Lippmann and Fein 1981). Tillie-Leblond and colleagues (2006) supported the findings of Lipmann and Fein with a drop in $\mathrm{PaCO}_{2}$ of $5 \mathrm{mmHg}$ or more as statistically indicative of concomitant PE (Tillie-Leblond et al 2006). On the other hand, Lesser and colleagues (1992) reported 108 patients presenting with a history of COPD and newly diagnosed PE to have no difference in the A-a gradient or reduction in $\mathrm{PaCO}_{2}$, despite prior hypercapnea on arterial blood gas (Lesser et al 1992). Hypoxemia remained a shared blood gas finding nonspecific to either disease. The correction of hypoxemia with oxygen or positive pressure flow may support the diagnosis of COPD, while major pulmonary embolism with intrapulmonary or intracardiac shunt may be resistant to correction (Pineda et al 2001).

\section{D-dimer}

In conjunction with pretest probability, the D-dimer is the preferred diagnostic tool in the outpatient setting and emergency department for DVT and PE. A low pretest probability and negative D-dimer effectively excludes thromboembolic disease. Work by Hartmann and colleagues (2000) found similar distribution of $\mathrm{D}$-dimer results in 313 patients with and without COPD, suggesting COPD had no influence on the diagnostic accuracy of the test for thromboembolic disease. This also suggested there was no increased prevalence of elevated D-dimer with COPD exacerbation. Sohn and colleagues (2006) found the combination of a low pretest probability and negative D-dimer among subsets of patients with increased age, prior malignancy, heart failure, or COPD sufficient in ruling out PE in 98\% of all-comers. Missed diagnoses were most prevalent among subsets of patients with known malignancy (Sohn et al 2006). A systematic review found a negative D-dimer to be as diagnostically useful as a normal lung perfusion scan or negative duplex ultrasonography in the setting of low pretest probability (Stein et al 2004).

\section{B-type natriuretic peptide}

B-type natriuretic peptide may be elevated in any disorder, causing right ventricular dilatation including acute PE, COPD, pulmonary hypertension with corpulmonale, or left-to-right shunting, causing ventricular overload. Such elevations are usually smaller than those appreciated in left ventricular heart failure and negligible or absent in uncomplicated COPD exacerbation (Bando et al 1999).

In the setting of PE, brain natriuretic peptide (BNP) has served more as a prognostic tool relating severity of 
right ventricular dysfunction to outcome than a diagnostic measure of PE presence. Kucher and colleagues (2003) found increased morbidity and mortality with elevated BNP and PE in patients without history of heart disease. Those needing urgent triage to more acute settings or dying shortly after their presentation had higher overall BNP levels (Kucher et al 2003). Kruger and colleagues (2004) studied 50 consecutive patients presenting with confirmed $\mathrm{PE}$ and found those without right ventricular dysfunction to have significantly lower BNP levels. A cutoff greater than 90 pg/ml was associated with a risk ratio of 28.4 for right ventricular dysfunction, though BNP was not predictive of mortality or in-hospital complication in this particular study (Kruger et al 2004).

\section{Troponin}

With uncomplicated COPD, troponin values are negative and helpful in ruling out acute cardiac ischemia as a cause of respiratory decompensation. Very rarely is there myocyte damage, except with severe respiratory failure and related poor oxygenation and ischemia.

With PE, right ventricular dilatation increases wall tension and decreases endocardial perfusion promoting myocardial damage. Giannitsis and colleagues (2000) reported the presence of troponin values greater than $0.1 \mathrm{ng} / \mathrm{ml}$ as indicative of right ventricular dysfunction associated with higher morbidity and mortality. Meyer and colleagues (2000) proposed that nearly a third of patients presenting with clinically diagnosed pulmonary embolism have elevated troponoin - I values. This was significantly associated with right ventricular dilatation and dysfunction as seen on echocardiography (Meyer et al 2000). Elevated troponin is absent in the setting of uncomplicated COPD, but if present, an evaluation for underlying $\mathrm{PE}$ is appropriate, especially when there is absence of signs and symptoms of acute ischemia.

\section{Lower extremity doppler ultrasonography}

The majority of pulmonary emboli (87\%-97\%) originate from the lower extremities, particularly the deep veins of the thigh. A study by Winter and colleagues (1983) of 29 patients with COPD exacerbation found 13 with DVT, two of which were later found to have PE postmortem (Winter et al 1983). Of these patients, none presented with clinical signs of DVT. Erelel and colleagues (2002) followed 56 patients with hospitalized COPD and detected DVT by ultrasound in eight. Five were subsequently diagnosed with pulmonary embolism by high probability V/Q scanning (Erelel et al 2002). Ambrosetti and colleagues (2003) suggested 10\% of patients with COPD have concomitant DVT, though this number may far underestimate actual prevalence. The risk of DVT appears increased due to poor mobility with worsening respiratory status, chronic steroid use, and active smoking (Erelel et al 2002). Treatment guidelines for inpatient COPD exacerbations currently recommend the standard use of DVT prophylaxis (GOLD et al 2005).

\section{$\mathrm{V} / \mathrm{Q}$ scan}

$\mathrm{V} / \mathrm{Q}$ scanning remains the imaging study of choice for diagnosing PE in patients who cannot tolerate a dye load because of acute or chronic kidney disease. Unfortunately, intrinsic lung processes such as pulmonary edema, pneumonia or anatomical perfusion, and ventilation defects confound the presence of thromboembolism. Lesser and colleagues (1992) found $60 \%$ of scans in the setting of COPD were of intermediate probability, with $22 \%$ later diagnosed with PE by angiography. High probability scans were positive for PE (5 of 5) while low probability scans did not have similar negative predictive values as compared with a general population because of increased pretest probability (Lesser et al 1992). Hartmann and colleagues (2000) concluded in a study of 509 patients with and without COPD that PE prevalence was similar between the two groups when V/Q was nondiagnostic and required angiographic confirmation. An increased frequency of high and low probability scans were found compared with Lesser's study. The utility of V/Q scanning in COPD appears diminished by a trend towards intermediate probability readings.

\section{Spiral CT/angiography}

Spiral chest CT and conventional angiography, the gold standards for PE diagnosis, were found by Hartmann and colleagues (2000) to have the same diagnostic performance in patients with and without COPD. Prevalence of PE appeared similar to other patient populations, suggesting equivalent sensitivity of the test and absence of association between PE and COPD.

\section{Summary and recommendations}

In studies evaluating PE and COPD, the presence of PE may be one in four among patients presenting with COPD exacerbation, a rate similar to a diagnosis of one in five among all patients presenting with acute dyspnea and a high pretest probability (Hoellerich and Wigton 1986; Wells et al 1998; Mispelaere et al 2002; Tillie-Leblond et al 2006). A careful evaluation of the epidemiology of both diseases finds the prevalence of PE in COPD similar to that of other disease 
population groups, and increased combined morbidity and mortality is similar to that of COPD and heart failure, or COPD and myocardial infarction (Incalzi et al 1997; Groenewegan et al 2003; Lee et al 2003; Holguin et al 2005; Huiart et al 2005; Sin et al 2006). Lesser and Hartmann's work confirmed that PE does not appear to favor COPD (Lesser et al 1992; Hartmann et al 2000). Nonetheless, morbidity and mortality is increased at one year and while diagnosis is difficult, an appreciation of the known morbidity of untreated thromboembolic disease makes acute detection of PE in the setting of COPD exacerbation important for improving clinical outcomes.

Findings of PE prevalence in COPD are also helpful in the clinical setting when attempting to make evidence-based decisions for diagnosis and management. The current literature suggests a one in four prevalence of $\mathrm{PE}$ in patients with atypical COPD exacerbation (Mispelaere et al 2002; TillieLeblond et al 2006). Twenty percent prevalence translates to at least $20 \%$ pretest probability in this patient group, which can be in addition to probabilities obtained by other clinical decision criteria, such as Wells or the Geneva score. The presumptive prevalence of $\mathrm{PE}$ in all COPD patients with infectious features is approximately $3 \%$ as found by Rutschmann and colleagues (2007) while DVT prevalence is estimated at $10 \%$ among all COPD patients according to Ambrosetti and colleagues (2003). Since DVT and PE are on the same spectrum of thromboembolic disease, accounting for DVT will increase the pretest probability of thromboembolic disease in those presenting with atypical exacerbations to a baseline of $30 \%$, and those with typical features to $13 \%$. Likelihood ratios for specific diagnostic tests can now be applied to obtain posttest probabilities for diagnosis of PE. As an example, Hartmann and colleagues (2000) found that $\mathrm{V} / \mathrm{Q}$ perfusion scanning in patients with COPD yielded a $79 \%$ specificity and $92 \%$ sensitivity when the test was interpreted as high probability, translating to a positive likelihood ratio of 4.38 \{ sensitivity/( 1 - specificity $)\}$. Patients with atypical COPD exacerbation whom underwent V/Q perfusion scanning would have a posttest probability of $65 \%$, while those with typical exacerbations would yield a posttest probability of $40 \%$, doubling in both cases our initial pretest probability, but not enough to rule in a positive diagnosis. This confirms again the finding that perfusion scanning, even when interpreted as high probability for PE in the setting of COPD exacerbation, is a poor diagnostic choice in either patient population when clinical suspicion is low.

In review, very little on physical exam delineates COPD and $\mathrm{PE}$ in patients with acute respiratory distress. Findings suggestive of underlying thromboembolic disease include dyspnea, absence of fever or sputum change, and tachycardia. Historical features such as prior DVT or PE, malignancy or decreased mobility are helpful and contributory to pretest probability. Hypoxemia is notable and nondiagnostic, with rales and wheezes overlapping both diseases. While suspicion of PE in patients presenting with noninfectious COPD exacerbation is intuitive, further cohort studies looking directly at the presence of PE in patients with typical infectious features are needed to reveal further underlying prevalence.

Laboratory and imaging studies in conjunction with clinical features contribute to pretest probability for PE. Though the presence of mildly elevated troponin and BNP in the setting of typical COPD exacerbation may provide clues as to underlying embolic disease, these values may be confounded by right or left ventricular failure and acute ischemia, all possible sequelae of advanced obstructive lung disease. Appropriate echocardiography and serial troponins to rule out ischemia would be warranted. Echocardiography may help delineate right vs. left ventricular dysfunction and diastolic failure contributing to an elevated BNP. The arterial blood gas is nonspecific and improved hypercapnea or hypoxemia with treatment is helpful in supporting uncomplicated COPD. A decrease in $\mathrm{PaCO}_{2}$ on initial presentation in the setting of prior hypercapnea with known COPD has been advocated as an indicator of underlying thromboembolism (Lippmann and Fein 1981; Tillie-Leblond et al 2006). It appears that patients with COPD develop DVT at rates similar to patients in a medical intensive care unit (Pomper and Luchman 1991; Kummer 1998; Ambrosetti et al 2003). Knowing this, and the fact that D-dimer distribution is similar in patients with and without COPD, makes it a useful test in conjunction with low pretest probability for ruling out thromboembolism. While management does not differ in sub-massive PE and DVT, PE remains more difficult to diagnose, and obtaining lower extremity Doppler ultrasound may clinch thromboembolic diagnosis faster than more extensive studies. V/Q scans are the default imaging study in the setting of acute or chronic kidney disease, but are diminished by increased frequency of nondiagnostic intermediate probability findings with COPD. Spiral CT has delineated similar prevalence of PE in patients with and without COPD, suggesting equivalent sensitivity of the test in both populations.

Figure 1 suggests a diagnostic algorithm for respiratory decompensation in patients with known COPD. The approach utilizes clinical prediction criteria for PE, such as the Wells criteria or Geneva score, to obtain a pretest probability and 


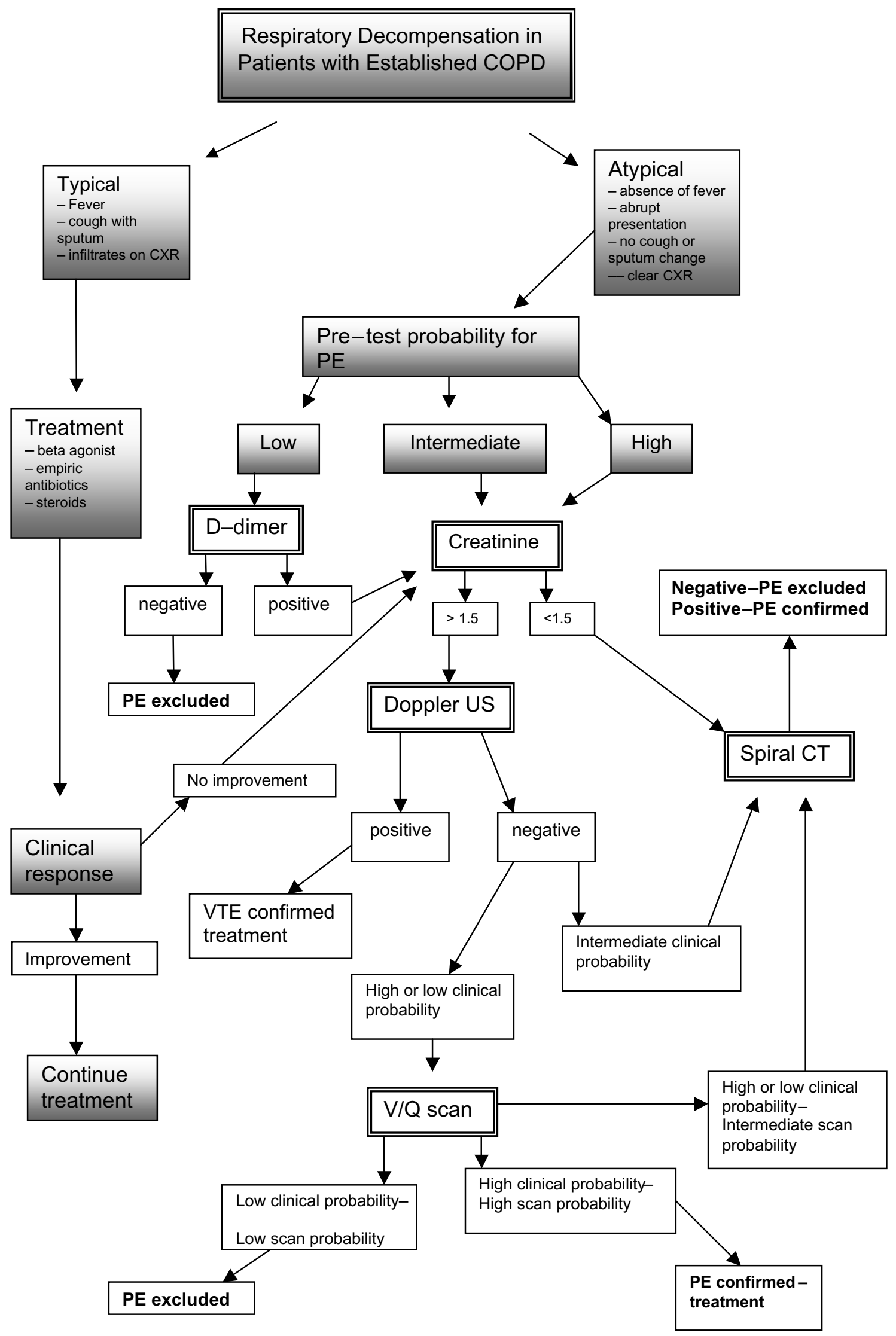

Figure I Respiratory decompensation in patients with established COPD.

Abbreviations: COPD, chronic obstructive pulmonary disease; $\mathrm{PE}$, pulmonary embolism; $\mathrm{V} / \mathrm{Q}$, ventilation/perfusion ratio. 
combines this with features of COPD exacerbation. Clinical history, physical exam, and laboratory data provide the impetus for pursuing PE diagnosis. In the absence of typical symptoms such as productive cough, fever, or decreased breath sounds diffusely, obtaining laboratory and diagnostic studies such as D-dimer, B - type natriuretic peptide, troponin, and arterial blood gas may be helpful in defining other underlying pathologies. Similarly, a nonresponse to aggressive COPD treatment with beta agonists, antibiotics, and steroids in patients with typical presentations supports evaluation for other causes of dyspnea.

Suspicion for PE in the setting of atypical COPD exacerbation can be divided into low, intermediate, or high pretest probabilities. Obtaining appropriate imaging beginning with assessment of serum creatinine is recommended as a first step. Where creatinine is less than $1.5 \mathrm{mg} / \mathrm{dl}$ and suspicion is intermediate or high, direct evaluation by spiral CT or angiography is the preferred confirmatory test. If there is low pretest probability, direct evaluation by spiral CT or angiography may not be appropriate, particularly if acute or chronic kidney disease precludes possibly nephrotoxic dye load. In this setting, lower extremity Doppler ultrasound and V/Q scanning may be helpful in establishing or ruling out diagnosis without risk of kidney injury. A low pre - test probability with a low D-dimer effectively rules out thromboembolism. Following the algorithm, high or intermediate pre - test probability and negative perfusion scanning or lower extremity Dopplers still warrants spiral CT or angiography. Often times, particularly in the setting of severe or advanced clinical picture, it may be necessary to forgo the possibility of false negative tests to obtain more definitive diagnostic imaging, so that treatment may be started in a timely fashion. Protecting the kidneys as much as possible from contrast nephropathy with standard pharmacological regimens is recommended.

Again, there is little general data evaluating the prevalence of PE in lone COPD with typical infectious features, making screening for PE on initial presentation unsupported. Rutschmann and colleagues (2007) argue against systematic screening in the setting of COPD exacerbation due to their findings of low prevalence in all-comers. DVT prevalence though is suggested to be as high as $10 \%$ in all patients with COPD, and in conjunction with clinical suspicion, Doppler studies may be the appropriate first imaging study to obtain if there is concern for thromboembolic diagnosis particularly if respiratory symptoms are equivocal.

In summary, patients with COPD exacerbation appear to experience sub-massive $\mathrm{PE}$ at rates similar to all patients presenting with dyspnea, as well as a high clinical suspicion for thromboembolism. Review of diagnostic laboratory and imaging studies as described above suggests an algorithm that incorporates appropriate pretest probability, laboratory studies such as D-dimer with emphasis on Doppler ultrasonography and CT angiography over perfusion scanning. Diagnosis of PE in COPD exacerbation is diminished by clinical difficulty, but incorporating thromboembolism into the differential may mean improved outcomes in COPD patients presenting with dyspnea and occult emboli.

\section{References}

Ambrosetti M, Ageno W, Spanevello A, et al. 2003. Prevalence and prevention of thromboembolism in patients with acute exacerbations of COPD Thromb Res, 112:203-7.

Bando M, Ishii Y, Sugiyama Y, et al. 1999. Elevated plasma brain natriuretic peptide levels in chronic respiratory failure with cor pulmonale. Respir Med, 93:507-14.

Carson J, Terrin ML, Duff A, et al. 1996. Pulmonary embolism and mortality in patients with COPD. Chest, 110:1212-19.

Cushman M, Tsai AW, White RH, et al. 2004. Deep venous thrombosis and pulmonary embolism in tow cohorts: the longitudinal investigation of thromboembolism etiology. Am J Med, 117:19-25.

Erelel M, Cuchadaroglu C, Ece T, et al. 2002. The frequency of deep venous thrombosis and pulmonary embolus in acute exacerbation of chronic obstructive pulmonary disease. Respir Med, 96:515-18.

Giannitsis E, Muller-Bardorff M, Kurowski V, et al. 2000. Independent prognostic value of cardiac troponin $\mathrm{T}$ in patients with confirmed pulmonary embolism. Circulation, 102:211-17.

[GOLD] Global Initiative for Chronic Obstructive Lung Disease, National Heart, Lung and Blood Institute, World Health Organization. 2005. Global strategy for the diagnosis, management, and prevention of chronic obstructive pulmonary disease. Bethesda (MD): 2005 Guideline, $115 \mathrm{pp}$ National Guidelines Clearinghouse.

Groenewegan KH, Schols AM, Wouters EFM. 2003. Mortality and mortality-related factors after hospitalization for acute exacerbation of COPD. Chest, 124:459-67.

Hartmann IJC, Hagen PJ, Melissant CF, et al. 2000. Diagnosing acute PE: Effect of COPD on the performance of D-dimer Testing, V/Q Scintigraphy, spiral CT angiography, and conventional angiography. Am J Respir Crit Care Med, 162:2232-37.

Hoellerich VL, Wigton RS. 1986. Diagnosing pulmonary embolism using clinical findings. Arch Intern Med, 146:1699-704.

Holguin FH, Folch E, Redd SC, et al. 2005. Comorbidity and mortality in COPD - related hospitalizations in the United States 1979-2001. Chest; 128:2005-11.

Huiart L, Ernst P, Suissa S. 2005. Cardiovascular morbidity and mortality in COPD. Chest, 128:2640-46.

Incalzi RA, Fuso L, De Rosa M, et al. 1997. Co - morbidity contributes to predict mortality of patients with chronic obstructive pulmonary disease. Eur Respir J, 10:2794-800.

Kozak LJ, Owings MF, Hall MJ. 2005. National hospital discharge survey: 2002 annual summary with detailed diagnosis and procedure data. Vital Health Stat 13, 158:1-199.

Kruger S, Graf J, Merx MW, et al. 2004. Brain natriuretic peptide predicts right heart failure in patients with acute pulmonary embolism. Am Heart J, 147:60-65.

Kucher N, Printzen G, Goldhaber SZ. 2003. Prognostic role of brain natriuretic peptide in acute pulmonary embolism. Circulation, 107:2545-47.

Kummer F. 1998. Patients with COPD: Their veins and pulmonary embolization. Respiration, 65:171-72.

Lee DS, Austin PC, Rouleau JL, et al. 2003. Predicting mortality among patients hospitalized for heart failure. JAMA, 290:2581-87. 
Lesser BA, Leeper KV, Stein PD, et al. 1992. The diagnosis of acute pulmonary embolism in patients with COPD. Chest, 102:17-22.

Lippmann M, Fein A. 1981. Pulmonary embolism in the patient with COPD: A diagnostic dilemma. Chest, 79:39-42.

Meyer T, Binder L, Hruska N, et al. 2000. Cardiac troponin I elevation in acute pulmonary embolism is associated with right ventricular dysfunction. J Am Coll Cardiol, 36:1632-36.

McGee, S. 2001. Evidence-based physical diagnosis. Philadelphia: Saunders.

Mispelaere D, Gierant JC, Audebert M, et al. 2002. Pulmonary embolism and sibilant types of chronic obstructive pulmonary disease decompensations. Rev Mal Respir, 19:415-23.

Pineda LA, Hathwar VS, Grant BJB. 2001. Clinical suspicion of fatal pulmonary embolism. Chest, 120:791-95.

Pomper SR, Luchman G. 1991. The role of intra-caval filters in patients with COPD and DVT. Angiology, 42:85-9.

Prescott SM, Richards KL, Tikoff G, et al. 1981. Venous thromboembolism in decompensated chronic obstructive pulmonary disease: A prospective study. Am Rev Respir Dis, 123:32-6.

Rutschmann OT, Cornuz J, Poletti PA, et al. 2007. Should pulmonary embolism be suspected in exacerbation of chronic obstructive pulmonary disease? Thorax, 62:121-5.

Sharma GVRK, Sasahara AA. 1975. Diagnosis of pulmonary embolism in patients with chronic obstructive pulmonary disease. J Chronic Dis, $28: 253-7$
Sin DD, Anthonisen NR, Soriano JB, et al. 2006. Mortality in COPD: role of comorbidities. Eur Respir J, 28:1245-57.

Sohn M, Kruip MJ, Nijkeuter M, et al. 2006. Accuracy of clinical decision rule, D-dimer, and spiral computated tomography in patients with malignancy, previous venous thromboembolism, COPD or heart failure in older patients with suspected pulmonary embolism. $J$ Thromb Hemost, 4:1042-6.

Stein PD, Hull RD, Patel KC, et al. 2004. D-dimer for the exclusion of acute venous thrombosis and pulmonary embolism: a systematic review. Ann Intern Med, 140:589-602.

Stein PD, Terrin ML, Hales CA, et al. 1991. Clinical, laboratory, roentgenographic, and electrocardiographic findings in patients with acute pulmonary embolism and no preexisting cardiac or pulmonary disease. Chest, 100:598-603.

Tillie-Leblond I, Marquette CH, Theirry P, et al. 2006. Pulmonary embolism in patients with unexplained exacerbation of COPD: Prevalence and risk factors. Ann Intern Med, 144:390-6.

Wells PS, Ginsberg JS, Anderson DR, et al. 1998. Use of a clinical model for safe management of patients with suspected pulmonary embolism. Ann Intern Med, 129:997-1005.

Winter JH, Buckler PW, Bautista AP, et al. 1983. Frequency of venous thrombosis in patients with an exacerbation of chronic obstructive lung disease. Thorax, 38:605-8. 